\title{
Philadelphia chromosome-positive acute lymphoblastic leukemia: a case report
}

\author{
Xingbin Dai ${ }^{1,2 \#}$, Fang Tian ${ }^{2,3 \#}$, Zuqiong $\mathrm{Xu}^{1,2 \#}$, Xiangtu Kong ${ }^{1,2}$, Pengjun Jiang ${ }^{1,2}$, Wen Xia ${ }^{1,2}$, \\ Xuejun Zhu ${ }^{1,2}$ \\ ${ }^{1}$ Department of Hematology, Jiangsu Province Hospital of Chinese Medicine, Affiliated Hospital of Nanjing University of Chinese Medicine, \\ Nanjing, China; ${ }^{2}$ First School of Clinical Medicine, Hospital of Nanjing University of Chinese Medicine, Nanjing, China; ${ }^{3}$ Research Center of \\ TCM, Jiangsu Province Hospital of Chinese Medicine, Affiliated Hospital of Nanjing University of Chinese Medicine, Nanjing, China \\ "These authors contributed equally to this work. \\ Correspondence to: Xuejun Zhu. Department of Hematology, Jiangsu Province Hospital of Chinese Medicine, No. 155 Hanzhong Road, Nanjing \\ 210029, China. Email: Zhuxj2@sina.com.
}

\begin{abstract}
Prognosis of patients with Philadelphia-positive acute lymphoblastic leukemia (Ph-ALL) relapsing after allogeneic hematopoietic stem cell transplantation (HSCT) is extremely poor. Therefore, effective alternative therapeutic measures are urgently needed. Recently, the use of antigen receptormodified T cells holds great promise for relapsed and refractory ALL treatment. Prior to chimeric antigen receptor T-cell (CAR-T) infusion conditioning chemotherapy is used routinely to establish a favorable in vivo environment for CAR-T expansion, which has mostly involved fludarabine and cyclophosphamide. We report on a patient presented with extreme fatigue and anemia and was diagnosed with relapsed and refractory acute lymphoblastic leukemia (ALL) harbored T315I-BCR-ABL mutation, who had undergone allogeneic HSCT and multiple reinducing chemotherapy, but achieved complete hematologic remission (CHR) with CAR - $\mathrm{T}$ infusion as a later salvage treatment. Prior to CAR-T infusion there was no conditioning chemotherapy, but a bone marrow suppression period induced by ponatinib. CAR-T cell infusion was well tolerated and the patient achieved a CHR and maintained it for three months. At present, there is no relevant report on the use of tyrosine kinase inhibitors (TKI) as preconditioning protocols before CAR-T cells infusion. Our case indicated ponatinib not only reduces tumor burden but may also serve as a conditioning regimen for CAR-T therapy in the treatment of relapsed and refractory Ph-ALL.
\end{abstract}

Keywords: Tyrosine kinase inhibitor (TKI); conditioning regimen; chimeric antigen receptor T-cell (CAR-T); case report; acute lymphoblastic leukemia (ALL)

Submitted Feb 22, 2020. Accepted for publication Jul 01, 2020.

doi: 10.21037/apm-20-469

View this article at: http://dx.doi.org/10.21037/apm-20-469

\section{Introduction}

Acute lymphoblastic leukemia (ALL) is an aggressive hematologic malignancy characterized by precursor lymphoblasts, blocked at an early stage of differentiation, proliferate rapidly and supplant normal hematopoietic cells in bone marrow. Over recent decades, there has been a significant improvement in ALL treatment outcome, which is due to such factors as intensive chemotherapy, targeted therapy with monoclonal antibodies and increased use of allogeneic hematopoietic stem cell transplantation (HSCT). However, the prognosis for adults with ALL remains significantly inferior to that for childhood patients. Adults with B-ALL have a much worse outcome and survival rates decline with age, to as low as $10-20 \%$ in the elderly (1). Prognosis of patients with relapsed and/or refractory ALL ( $\mathrm{r} / \mathrm{r}$ ALL) is particularly poor, with most studies reporting 5 -year overall survival rates of $\leq 10 \%(2,3)$. Besides, Philadelphia chromosome-positive represents the most unfavorable prognosis among leukemia, which 
increases with age. Research shows that the incidence rate of Philadelphia chromosome was as high as $50 \%$ in adults older than 60 years with B-cell ALL $(4,5)$. Therefore, the treatment of adults with $\mathrm{r} / \mathrm{r}$ prognosis of patients with Philadelphia-positive acute lymphoblastic leukemia ( $\mathrm{Ph}-$ ALL) has been proven more challenging.

Recently, chimeric antigen receptor T-cell (CAR-T) therapy has shown great promise in the advancement of individualized clinical cancer immunotherapy, which has been successfully applied in the treatment of B-cell malignancies. Novartis' Kymriah (tisagenlecleucel) became the first FDA-approved CAR-T therapy in the treatment of relapsed or refractory B-cell ALL in the United State in 2018, highlighting the success of CAR-T cell-based immunotherapy (6). However, there are still some problems needing an in-depth discussing, including pre-collection therapy, conditioning therapy, and therapy resistance. Here we report on a Ph-ALL patient who relapsed early after allogeneic HSCT and harbored T315I-BCR-ABL mutation, but achieved complete hematologic remission (CHR) with CAR-T infusion as a later salvage treatment. Prior to CAR-T infusion there was no conditioning chemotherapy, but a bone marrow suppression period induced by ponatinib, which is unique about this case. CAR-T cell infusion was well tolerated and the patient achieved a CHR and maintained it for three months. Our data showed that the tyrosine kinase inhibitor (TKI) can be used as a part of conditioning regimens in the treatment of $\mathrm{r} / \mathrm{r}$ ALL with T315I BCR-ABL mutation in CAR-T therapy. We present the following article in accordance with the CARE reporting checklist (available at http://dx.doi. org/10.21037/apm-20-469).

\section{Case presentation}

A 56-year-old woman who presented at a local hospital with fatigue in June, 2014. Physical examination found abnormality. There was no abnormal in her medical history, family, and psycho-social history. Complete blood cell analysis showed that a white blood cell (WBC) count of $39.6 \times 10^{9} / \mathrm{L}$ (normal range, 4 to $10 \times 10^{9} / \mathrm{L}$ ), hemoglobin $(\mathrm{Hb})$ of $81 \mathrm{~g} / \mathrm{L}$ (normal range, 120 to $150 \mathrm{~g} / \mathrm{L}$ ), and platelet (PLT) of $17 \times 10^{9} / \mathrm{L}$ (normal range, 100 to $300 \times 10^{9} / \mathrm{L}$ ). A bone marrow aspirate (BMA) revealed $85 \%$ lymphoblasts. Flow cytometry showed blasts expressing CD7, CD34, CD19, CD33, TDTc, and CD79 $\alpha$. A positive p190 BCR-ABL (ela2) transcript was revealed by QRT-PCR. Therefore, the patient was diagnosed definitely with Ph-positive ALL
(Normal B cell type, poor prognosis group). She received induction chemotherapy with idarubicin (12 $\mathrm{mg}, \mathrm{d} 1-3)$, vincristine $(2 \mathrm{mg}, \mathrm{d} 1,8,15,22)$, dexamethasone $(50 \mathrm{mg}$, d1-14, $20 \mathrm{mg}, \mathrm{d} 15-28)$ and PEG-asparaginase (2,500 IU, $\mathrm{d} 1,15)$ on June 10, 2014. She developed hematologic grade 4 toxicities according to the National Cancer Institute Common Terminology Criteria for Adverse Events version 4.0 (CTCAE), septicemia and heart failure after the first course of chemotherapy. He was supported with blood transfusion, potent antibiotics and promoted the heart function with subsequent recovery. She was then discharged after hematopoietic recovery and was given oral administration of imatinib mesylate capsules $(0.4 \mathrm{~g} /$ day $)$. Bone marrow examination revealed the patient obtained a CHR with complete cytogenetic response (CCyR) on August 14, 2014. She then received consolidation chemotherapy using VMCP (vindesine $2 \mathrm{mg} \mathrm{d} 1,8,15,22$, methotrexate $10 \mathrm{mg} \mathrm{d} 1-3$, cyclophosphamided $1.2 \mathrm{~g} \mathrm{~d} 1,8$, and hydroprednisone $40 \mathrm{mg} \mathrm{d} 1-10)$ in combination with imatinib $(0.4 \mathrm{~g} /$ day $)$. The patient continued to receive two consecutive consolidation courses in September and October respectively according to the COAP protocol (cyclophosphamide $0.6 \mathrm{~g} \mathrm{~d} 1,3$, vindesine $2 \mathrm{mg} \mathrm{d} 1$, cytarabine $200 \mathrm{mg} \mathrm{d} 1-7$ and hydroprednisone $60 \mathrm{mg} \mathrm{d1-7)}$ and lumbar puncture revealed the cerebrospinal fluid was negative. And then accepted another consolidation chemotherapy with idarubicin (10 $\mathrm{mg}, \mathrm{d} 1-3)$, vincristine (2 $\mathrm{mg} \mathrm{d} 1)$, cyclophosphamide $(0.6 \mathrm{~g}, \mathrm{~d} 1,3)$, dexamethasone $(10 \mathrm{mg} \mathrm{d} 1-$ 7) and PEG-asparaginase $(2,500 \mathrm{IU}, \mathrm{d} 1,14)$ on December 7, 2014. Review of bone marrow examination revealed the presence of 22\% lymphoblasts on January 20, 2015. G-band chromosome analysis showed $\mathrm{Ph}$ chromosome translocation $\mathrm{t}(9 ; 22)(\mathrm{q} 34 ; \mathrm{q} 11)$ in $100 \%$ of metaphases $(20 / 20$ cells) analyzed. She underwent reinduction chemotherapy (fludarabine, idarubicin, Ara-C, granulocyte colonystimulating factor) in combination with nilotinib and achieved CR. She underwent bone marrow transplantation (BMT) from a human leukocyte antigen (HLA)-identical sibling after conditioning with antithymocyte globulin, fludarabine and cyclophosphamide). No graft-versus-host disease (GVHD) occurred with cyclosporin (CsA) and shortterm dexamethasone as prophylaxis. Chimerism analysis displayed a mixed chimerism (87.32\% donor) on April 15,2015 . She remained in remission for 4 months before relapsing in May 2015. At that time, she had an elevated leukocyte count $\left(44 \times 10^{9} / \mathrm{L}\right)$. Bone marrow analysis revealed hypercellularity with significant myeloid hyperplasia with $61.0 \%$ myeloblasts. The persistence of Philadelphia 

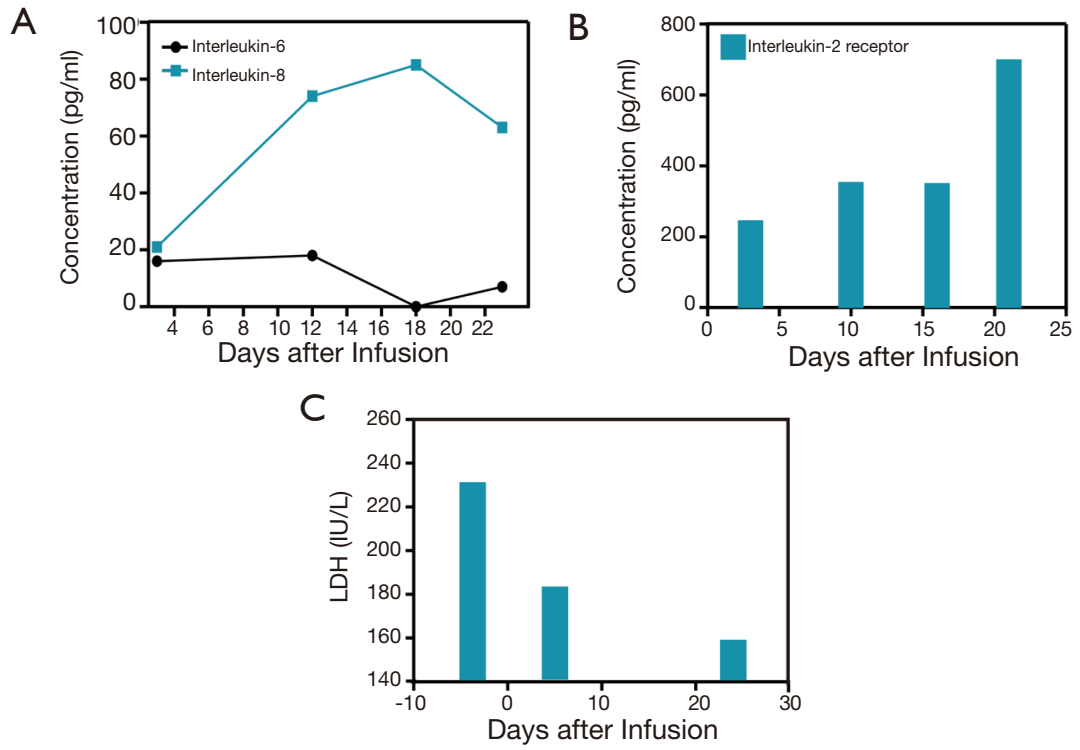

Figure 1 Change levels of cytokine levels and lactate dehydrogenase (LDH) after anti-CD19 CAR-T cell infusion. (A) Cytokine levels of interleukin-6, interleukin-8 increased significantly and reached the peak on day 18 after anti-CD19 CAR-T cell infusion; (B) interleukin-2 receptor increased significantly; (C) LDH was decreased gradually.

chromosome $(99.3 \% \mathrm{Ph}+)$ was identified by Fluorescence in situ hybridization (FISH) analysis. She then started receiving dasatinib treatment at a dose of $70 \mathrm{mg} /$ day. Two weeks later, she experienced grade 3 anemia, grade 4 neutropenia, and 2 thrombocytopenia according to CTCAE. BMA showed $26 \%$ lymphoblasts. She stopped taking dasatinib and peripheral blood cell steadily increasing. Early in July, 2015 , she had an elevated leukocyte count $\left(68.9 \times 10^{9} / \mathrm{L}\right)$ and a direct sequencing study revealed a T315I mutation of the BCR-ABL gene. Ponatinib treatment was initiated at a dose of $45 \mathrm{mg} /$ day. After 7 days of ponatinib therapy, she developed hematologic grade 4 toxicities and septicemia. She stopped taking ponatinib and was supported with blood transfusion, potent antibiotics with subsequent recovery. Since then, she received palliative treatment with dasatinib (15 mg, once daily). The subsequent BMA evaluation showed $1.0 \%$ lymphoblasts on July 27, 2015. Then severe granulocytopenia pressed her to stop taking dasatinib. On August 12, 2015, complete blood cell analysis showed that the $\mathrm{WBC}$ was $7.36 \times 10^{9} / \mathrm{L}, \mathrm{Hb}$ was $100 \mathrm{~g} / \mathrm{L}$ and PLT was $17 \times 10^{9} / \mathrm{L}$. Myeloblasts were not found in peripheral blood. Subsequently, $100 \mathrm{~mL}$ of peripheral blood mononuclear cells (PBMCs mL) were collected by use of blood corpuscle separator (COBE SPECTRA system) to prepare anti-CD19 CAR-T cells that had been expanded with anti-CD3 and anti-CD28 antibodies and lentivirally transduced to express the anti-CD19 CARs by reference to relevant literature $(7,8)$. The patient suffered a relapse with $60 \%$ blasts present in the bone marrow On August 15, 2015. Conditioning regimen with ponatinib (45 $\mathrm{mg} /$ day, $\mathrm{d}-7$ to $\mathrm{d}-2)$ was given on August 24, 2015. Then, anti-CD19 CAR-T cells were infused at a total dose of $3.0 \times 10^{6} \mathrm{CAR}-\mathrm{T}$ cells $/ \mathrm{kg}$ (transduction efficiency was more than $30 \%$ ) on August 26, 2015. No obvious adverse reactions occurred. Cytokine levels (interleukin-6, interleukin-8, interleukin-2 receptor) increased significantly (Figure 1A,B). Lactate dehydrogenase (LDH) was decreased gradually (Figure $1 C$ ). The hematopoietic recovery returned to normal completely on day+14 to day+15 (Figure 2). A transiently rapid expansion of Anti-CD19 CAR-T cells in the peripheral blood on day+6 was revealed and then gradually declined (Figure 3). On September 16, 2015 (day+12), the patient's bone marrow was proliferous and active in low level, and there was no blast cell (Figure 4A). Flow cytometry of bone marrow cells detected MRD negative (Figure 4B). Positive BCRABL p190 transcript (0.5\%) was detected by QRT-PCR. The chromosome karyotype was normal. Therefore, she achieved a complete remission. However, poor economic condition of family disinclined her to use ponatinib as a maintenance therapy or secondary allogeneic HSCT. Then follow-up was conducted every 2 weeks. Unfortunately, the patient had another recurrence with $23 \%$ bone marrow 

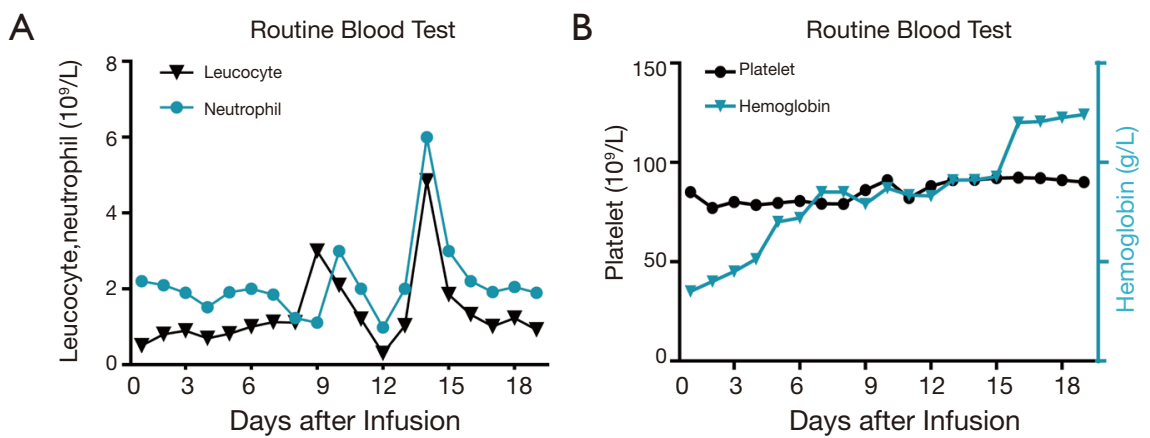

Figure 2 The hematological changes after anti-CD19 CAR-T cell infusion. Peripheral blood cells tended to stabilized on day 15 after antiCD19 CAR-T cell infusion.
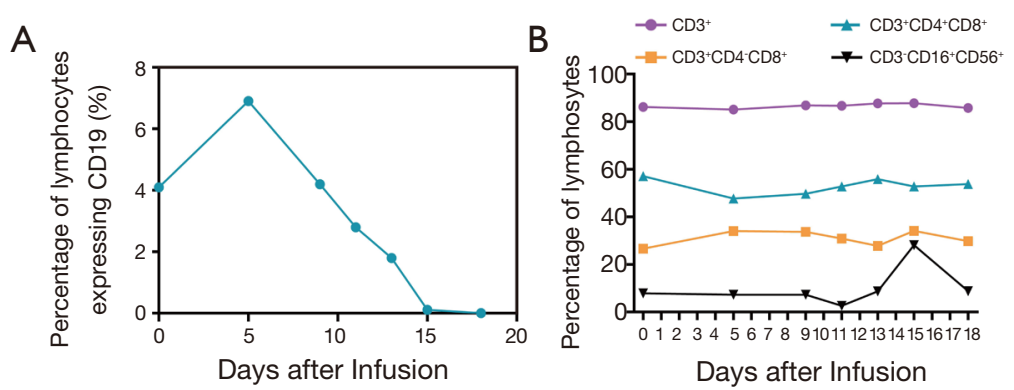

Figure 3 The percentage change of lymphocytes expressing CD19 and T lymphocytes after anti-CD19 CAR-T cell infusion. (A) The change curve of anti-CD19 CAR-T cells and T cell subgroup anti-CD19 CAR-T cells revealed transiently rapid expansion in the peripheral blood on day+6; (B) T cell subgroup achieved stability about one week after anti-CD19 CAR-T cell infusion.

blasts 88 days later. She abandoned treatments because of the economy and died at the end of November. Timeline of disease course is shown in Figure 1.

All procedures performed in studies involving human participants were in accordance with the ethical standards of the institutional and national research committee and with the Helsinki Declaration (as revised in 2013). Informed consent for the publication of the report, and the accompanying images, was provided by the patient's son. The submission version of the report was read by his son, and the report's content was confirmed as being correct to the best of his knowledge.

\section{Discussion}

In the present case, the patient was a Ph-ALL patient who relapsed early after allogeneic HSCT and harbored T315IBCR-ABL mutation, but achieved CHR with CAR-T infusion as a later salvage treatment, which was conducted in the period of myelosuppression caused by the third- generation TKI ponatinib without a conditioning regimen treatment.

There is no standard therapy for $\mathrm{r} / \mathrm{r} \mathrm{Ph}$-ALL after transplantation and clinical trials are recommended by National Comprehensive Cancer Network (NCCN) guideline. For this patient harboring T315I-BCR-ABL mutation, we hold the opinion that ponatinib was the only effective drug. The therapeutic response had proved that our case was very sensitive to ponatinib treatment. About three weeks later, a bone marrow aspirate revealed another complete hematologic response. The patient refused to continue ponatinib maintenance treatment or a second allogeneic HSCT because of the poor economy. At this point, our patient was recommended for CAR-T clinic trial. Unfortunately, she developed another ALL relapse 20 days after discontinuation of her ponatinib. The $\mathrm{T}$ cells are activated, transduced with the CAR transgene, expanded to the required cell numbers for therapy. After the production of autologous CAR-T cells was complete, we were serious about making conditioning chemotherapy in 
A
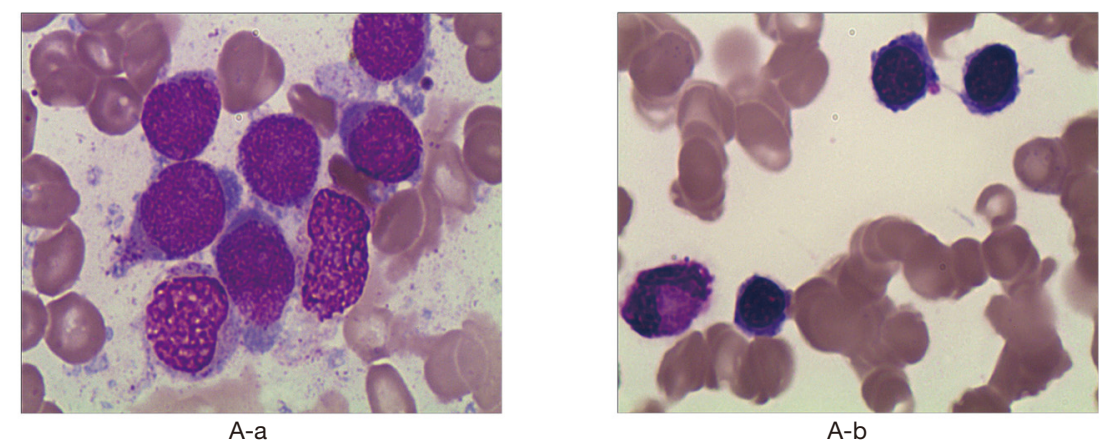

B
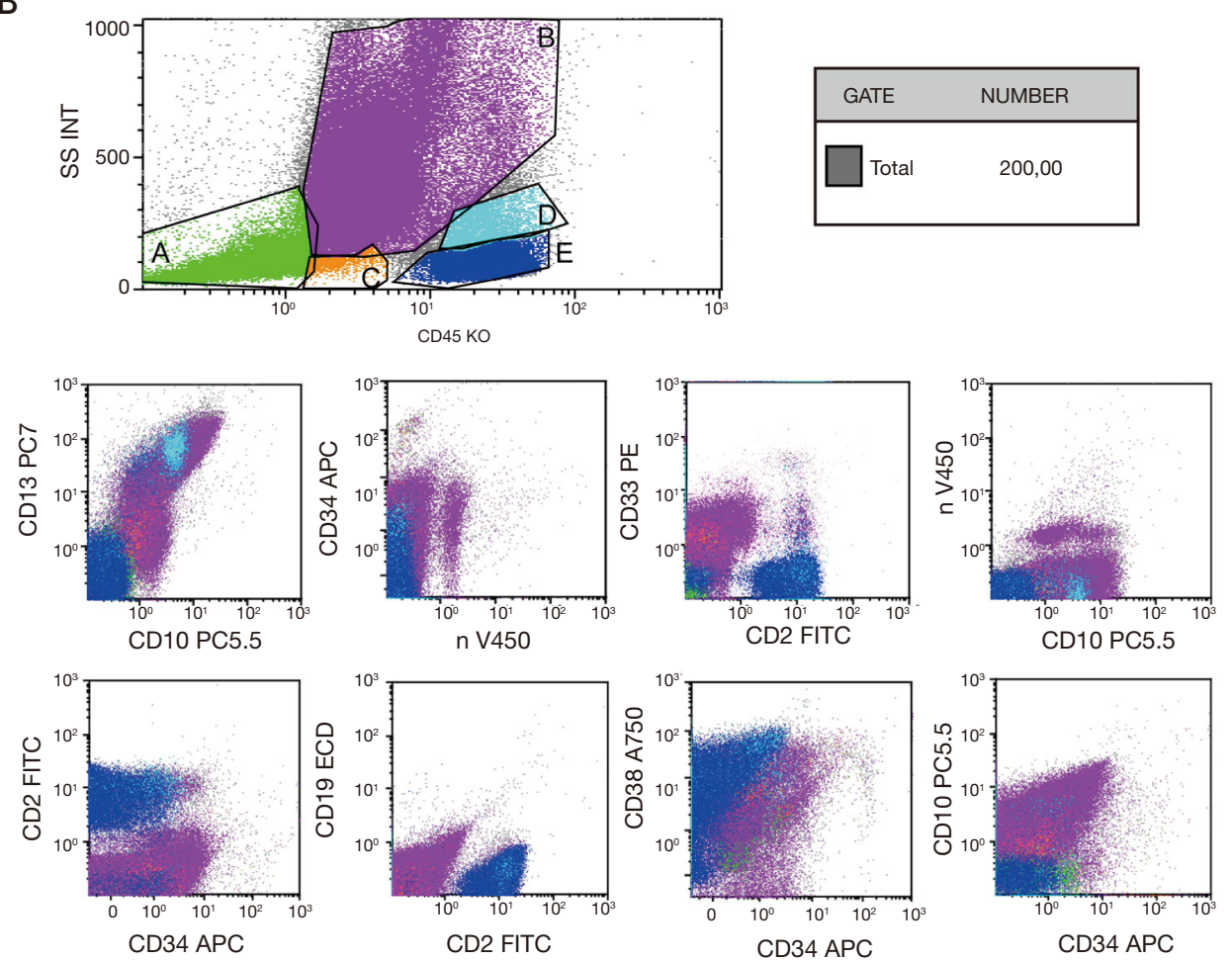

Figure 4 Examination of marrow cell was reviewed. (A-a) Hyperplasia of bone marrow was extremely active before receiving the treatment; (A-b) bone marrow was proliferous and active in low level. The cell metamorphosis was observed by Wright staining and at 1,000 times magnification; (B) flow cytometry of bone marrow cells detected MRD negative on day12 after anti-CD19 CAR-T cell infusion.

consideration of her multiple severe infections. At the same time, she was insensitive to conventional chemotherapeutic drugs and she showed the greatest fear of chemotherapy. Considering that she responds well to ponatinib and shortterm treatment with ponatinib was safe, we used ponatinib as an alternative to fludarabine and cyclophosphamide as the conditioning regimen. In order to establish a favorable in vivo environment for CAR-T expansion, CAR-T studies has mostly employed conditioning chemotherapy $1-3$ days prior to CAR-T infusion, with cyclophosphamide and fludarabine commonly chosen for their lymphocyte-depleting effects (9).
The inclusion of fludarabine reduces anti-murine CAR rejection and supports longer CAR-T cell persistence was also demonstrated (10). While there were some evident toxic side effects of fludarabine and cyclophosphamide, further optimization being explored, which may enhance expansion and persistence, while reducing toxicities (11).

TKI exerts profound immunomodulatory effects on $\mathrm{T}$ cells and antigen-presenting cells, such as dendritic cells (12), which plays major roles in immune tumor surveillance and the outcome of HSCT. TKI therapy may thus control cancer cell growth both directly and indirectly 
by changing the immunologic microenvironment (13). As a specific example of a treatment that TKI affects $\mathrm{T}$ cells, in chronic lymphocytic leukemia (CLL) the kinase inhibitor ibrutinib inhibits malignant $\mathrm{B}$ cell proliferation through BTK, but also affects non-malignant $T$ cells and shifts T helper cells towards the Th1 immune subset (14). Up till now, an emerging clinical goal is to use treatments that reduce tumor burden, enhance CAR-T cell expansion and persistence. Therefore, patients may receive therapy involved lymphodepleting chemotherapy, antibodies that have long half - lives or continuous oral therapies such as kinase inhibitors. The expansion changes of postinfusion CAR-T cells and complete remission turned out that TKI can be used as starting material for the manufactured CAR-T cells product and chosen for conditioning regimen prior to CAR-T cells infusion.

An increase in the frequency of CAR-T cells by day 3-5 and then a gradual decline was observed in our patient, which is consistent with previous report $(15,16)$. Although there was no direct evidence of the tumor cytokine release syndrome (CRS), other than changes in the LDH and IL-6 level, which is likely a central mediator of CRS after CAR-T cell therapy (17). More often than not the reason might be found in the following two aspects. The burthen of leukemia cells was minished after treatment with ponatinib. Our patient received a lower CAR-T cell doses. Factors influencing CRS include T cell engagement with the target antigen followed by proliferation and functional response, disease type, nature and degree of lymphodepletion, and possibly CAR design (17). Dasatinib as a second-generation TKI can halt cytolytic activity, cytokine production, and proliferation of CAR-T cells in vitro and in vivo (18). A short treatment course of dasatinib, administered early after CAR-T cell infusion, protects a proportion of mice from otherwise fatal CRS in a mouse model of CRS (18). This article also puts forward that further investigation into the influence of ponatinib on CAR-T cell activity is needed.

The above description of clinical course provides the first-known case of AML in complete remission receiving CAR-T cell therapy without regular conditioning regimen with cyclophosphamide or fludarabine. The reason why the clinical effects was not affected appears to be that the ponatinib treatment reduced leukemia cells loads and regulated the function of immunity, establishing a favorable in vivo environment for CAR-T expansion.

In this article, we can conclude that it is feasible to use ponatinib as a bridge to CAR-T therapy in patients with Philadelphia chromosome-positive ALL, At least, in the near term. But the long-term effect is uncertain, because the patient had no desire to continue treatment with ponatinib or CAR-T cell. Large-sample and multicenter studies should be preformed to further explore conditioning regimens that has the potential to modulate the $\mathrm{T}$ cells, enhance expansion and persistence, while reducing toxicities for CAR-T cells. The effect of the TKIs on the microenvironment in vivo and related mechanisms still needs further research.

\section{Acknowledgments}

Funding: This work was financially supported by the Science and Technology Development Program of Jiangsu Province -Clinical Frontier Technology (grant Nos. BE2016809) and the Nanjing Science and Technology Development Program (grant Nos. 201503011).

\section{Footnote}

Reporting Checklist: The authors have completed the CARE reporting checklist. Available at http://dx.doi.org/10.21037/ apm-20-469

Conflicts of Interest: All authors have completed the ICMJE uniform disclosure form (available at http://dx.doi. org/10.21037/apm-20-469). The authors have no conflicts of interest to declare.

Ethical Statement: The authors are accountable for all aspects of the work in ensuring that questions related to the accuracy or integrity of any part of the work are appropriately investigated and resolved. All procedures performed in studies involving human participants were in accordance with the ethical standards of the institutional and national research committee and with the Helsinki Declaration (as revised in 2013). Informed consent for the publication of the report, and the accompanying images, was provided by the patient's son. The submission version of the report was read by his son, and the report's content was confirmed as being correct to the best of his knowledge.

Open Access Statement: This is an Open Access article distributed in accordance with the Creative Commons Attribution-NonCommercial-NoDerivs 4.0 International License (CC BY-NC-ND 4.0), which permits the noncommercial replication and distribution of the article with the strict proviso that no changes or edits are made and the 
original work is properly cited (including links to both the formal publication through the relevant DOI and the license). See: https://creativecommons.org/licenses/by-nc-nd/4.0/.

\section{References}

1. Geyer MB, Hsu M, Devlin SM, et al. Overall survival among older US adults with ALL remains low despite modest improvement since 1980: SEER analysis. Blood 2017;129:1878-81.

2. Gregory S. Adult Acute Lymphoblastic Leukemia:Treatment and Management Updates. Semin Oncol Nurs 2019;35:150951.

3. Gökbuget N, Stanze D, Beck J, et al. Outcome of relapsed adult lymphoblastic leukemia depends on response to salvage chemotherapy, prognostic factors, and performance of stem cell transplantation, Blood 2012;120:2032-41.

4. Roberts KG, Gu Z, Payne-Turner D, et al. High frequency and poor outcome of Philadelphia chromosomelike acute lymphoblastic leukemia in adults. J Clin Oncol 2017;35:394-401.

5. Moorman AV, Chilton L, Wilkinson J, et al. A populationbased cytogenetic study of adults with acute lymphoblastic leukemia. Blood 2010;115:206-14.

6. Helsen CW, Hammill JA, Lau VWC, et al. The chimeric TAC receptor co-opts the $\mathrm{T}$ cell receptor yielding robust anti-tumor activity without toxicity. Nat Commun 2018;9:3049.

7. Hollyman D, Stefanski J, Przybylowski M, et al. Manufacturing validation of biologically functional $\mathrm{T}$ cells targeted to CD19 antigen for autologous adoptive cell therapy. J Immunother 2009;32:169-80.

8. Tumaini B, Lee DW, Lin T, et al. Simplified process for the production of anti-CD19-CAR engineered T cells. Cytotherapy 2013;15:1406-15.

9. Hay KA, Turtle CJ. Chimeric Antigen Receptor (CAR) T

Cite this article as: Dai $\mathrm{X}$, Tian $\mathrm{F}, \mathrm{Xu} Z$, Kong $\mathrm{X}$, Jiang $\mathrm{P}$, $\mathrm{Xia} \mathrm{W}, \mathrm{Zhu} \mathrm{X}$. Philadelphia chromosome-positive acute lymphoblastic leukemia: a case report. Ann Palliat Med 2021;10(1):742-748. doi: 10.21037/apm-20-469
Cells: Lessons Learned from Targeting of CD19 in B-Cell Malignancies. Drugs 2017;77:237-45.

10. Turtle CJ, Hanafi LA, Berger C, et al. CD19 CAR-T cells of defined CD4+:CD8+ composition in adult B cell ALL patients. J Clin Invest 2016;126:2123-38.

11. Jain MD, Davila ML. Engineering chimeric antigen receptor-T cells for cancer treatment. Stem Cells 2017;36:47-62.

12. Rohon P, Porkka K, Mustjoki S. Immunoprofiling of patients with chronic myeloid leukemia at diagnosis and during tyrosine kinase inhibitor therapy. Eur J Haematol 2010;85:387-98.

13. Seggewiss R, Price DA, Purbhoo MA. Immunomodulatory effects of imatinib and second-generation tyrosine kinase inhibitors on T cells and dendritic cells: an update. Cytotherapy 2008;10:633-41.

14. Dubovsky JA, Beckwith KA, Natarajan G, et al. Ibrutinib is an irreversible molecular inhibitor of ITK driving a Th1-selective pressure in T lymphocytes. Blood 2013;122:2539-49.

15. Zhang C, Kong PY, Li S, et al. Donor-derived CAR-T Cells Serve as a Reduced-intensity Conditioning Regimen for Haploidentical Stem Cell Transplantation in Treatment of Relapsed/Refractory Acute Lymphoblastic Leukemia: Case Report and Review of the Literature. J Immunother 2018;41:306-11.

16. Grupp SA, Kalos M, Barrett D, et al. Chimeric Antigen Receptor-Modified T Cells for Acute Lymphoid Leukemia. N Engl J Med 2013;368:1509-18.

17. Porter D, Frey N, Wood PA, et al. Grading of cytokine release syndrome associated with the CAR T cell therapy tisagenlecleucel. J Hematol Oncol 2018;11:35-47.

18. Mestermann K, Giavridis T, Weber J, et al. The tyrosine kinase inhibitor dasatinib acts as a pharmacologic on/off switch for CAR T cells. Sci Transl Med 2019;11:eaau5907. 